\title{
Virtual reality - level of immersion within the crime investigation
}

\author{
Václav Mach ${ }^{1}$, Jan Valouch ${ }^{1}$, Milan Adámek ${ }^{1}$, and Jiři Ševčík ${ }^{1 *}$ \\ ${ }^{1}$ Tomas Bata University in Zlín, Faculty of Applied Informatics, Nad Stráněmi 4511, 76005 Zlín, Czech Republic.
}

\begin{abstract}
The 3D reconstruction and visualization of crime scenarios, such as criminal assaults, traffic accidents or homicides are defining a new approach with potential for increasing effectiveness within the criminal investigation process. Modern 3D capturing and processing methods are utilized to create an authentic and immersive virtual environment. Nonetheless, an immersion to virtual environment enables different levels of view. Virtual reality immersion views which are proposed and described. These views are specific by reasons of its role within the criminal investigation as well as its technical severity. Moreover, the possible methods for the design of proposed virtual views are analyzed and the next research steps are exposed.
\end{abstract}

\section{Introduction}

Recent progress in virtualization of the real environment, including the scenarios that can possibly take place in it, draws the attention of both the professional and the scientific community. The application of virtual reality (VR) aspires to the post of reformer in terms of the natural connection of man and computer technology. VR could be defined as an advanced user interface that includes real-time simulation of the real environment where the user is interacting through several sensory channels: sight, hearing, touch, smell, and taste. The more human body sensors are involved, the more authentic is the VR experience. There are several research areas, where an application of advanced scanning and visualization techniques is examined such as health service, architecture, tactic simulators for purposes of army or police forces, etc. However, this article discusses an application from the forensic science area, particularly an extension of widely examined crime scene reconstruction topic.

\subsection{D crime scene reconstruction}

A variety of $3 \mathrm{D}$ recording techniques have been employed for the documentation, analysis, and presentation of a crime scene. 3D data capture methods provide precise scene recording that does not suffer from the spatial distortion effects associated with 2D photographic recording [1]. However, with the advent of equipment that is faster and easier to use, and the recent advances in software, police forces can now regularly capture and more importantly utilize 3D data for crime scene documentation [1]. Empirical research has investigated the accuracy of 3D scene recording, for example, a comparison of measurement data from two different methods for documenting crime scenes (a tape measure and a 360 camera with photogrammetry), found the manual method to be more accurate but the software application method more precise [2]. Further, 3D scan data may be combined with CCTV or eyewitness photographs, which can for example aid with identifying the spatial position of an individual or for profiling individuals (e.g. to estimate height) [1]. For larger areas, such as open fields and rooms within a building, groundbased laser scanners are suitable (terrestrial laser scanners). These scanners use either a pulsed (time of flight) or continuous frequency modulated (phase-shift) laser that measures a distance to the surface of an object [3].

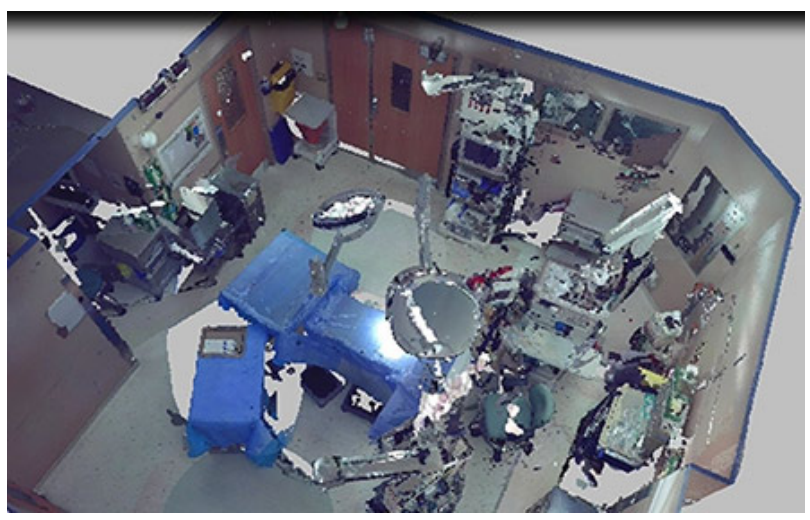

Fig. 1. Virtual crime scene reconstruction example.

The introduction of virtual animations is a novel area, which can depict changes over time, use zooming or animated subjects [4] and/or combine photography of real-world evidence (e.g. photographs of injuries) with virtually constructed scenes or figures [5]. A recent example used a virtual animation to depict how a victim obtained their injuries, through animating a moving 3D skeleton with bullet paths penetrating the body [6].

\footnotetext{
* Corresponding author: sevcik@utb.cz
} 
Furthermore, combining multimodal imaging with CAD has been used to show elaborate virtual representations and interactive videos of injuries and weapons [7].

\subsection{Courtroom display examination}

Another research related to crime investigation is aimed at an assessment of $3 \mathrm{D}$ visualization in courtroom display influence. The utility of 3D digital data for courtroom demonstrations of osteological evidence and the potential advantages of having $3 \mathrm{D}$ models over $2 \mathrm{D}$ photographs was detailed by Errickson et al. [8]. However, there is currently little data to show whether $2 \mathrm{D}$ or $3 \mathrm{D}$ exhibits are more effective or comprehensible, and similarly more or less prejudicial to a jury [4]. A recent report highlighted that research is needed to fully explain and quantify the utility of visual aids in a court of law [9]. Moreover, using VR in criminal trials poses new issues, an article by Young [10] described that VR demonstrations of evidence can be particularly persuasive and even prejudicial, and the author advised courts of law to proceed with due caution [10]. Contrastingly, Salmanowitz [11] suggested that VR could aid in reducing bias in courtroom decision making.

Kilgus et al. [12] provided caution for using mixed reality MR and Animations in courtrooms, the authors state the necessity for verifying the authenticity, fairness, and relevance of using of visualizations for courtroom display of evidence, noting that the data, methods and visualization must be valid. Virtual $3 \mathrm{D}$ models and physical 3D replicas have been used in courts of law as exhibits, there are high value and responsibility involved in courtroom display and several areas require clear definitions to be applied [13]. Firstly, it is important to differentiate between findings and simulated scenarios such as scene reconstructions [4]. A 3D crime scene reconstruction is based on factual scientific evidence, in comparison to a crime scene simulation, which based on a predicted condition or sequences of events [1]. The two must be distinguished when presenting $3 \mathrm{D}$ virtual scenes and practitioners have a responsibility to ensure that courts of law understand this distinction. Secondly, exhibits used as demonstrative aids or demonstrative evidence must be separated; each has different rules of admissibility and moreover, demonstrative aids are not admitted into evidence and carry no probative value [14, $15]$.

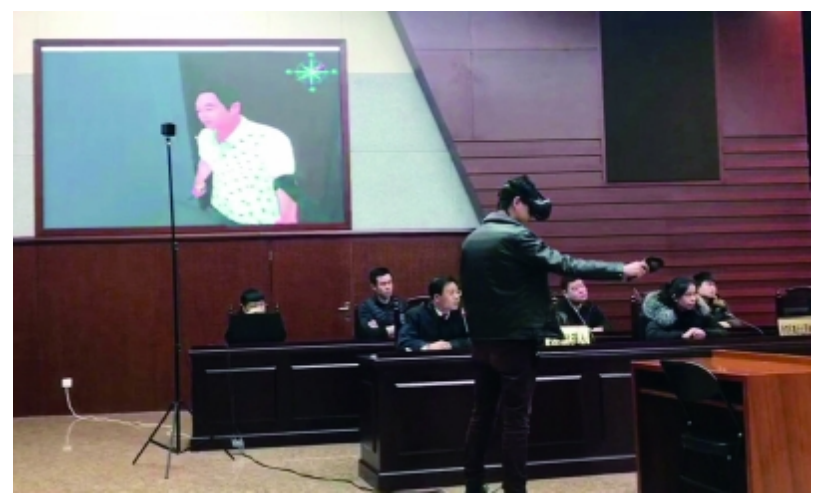

Fig. 2. Courtroom crime demonstration in China.

\subsection{Virtual crime scenario}

Recent grow in 3D capturing and visualization techniques, based on simultaneous evolution in computer performance finally opens possibilities of utilizable applications of VR. Although VR utilization within the crime scene investigation is still not proved as a finalized method with probative value, it's necessary to realize that $3 \mathrm{D}$ reconstruction and visualization techniques are still evolving. On the other hand, more complex will be process of crime scene interpretation, more effective the court can assess the whole situation. Moreover, the combination of standard approach with modern possibilities seems to like an appropriate start.

The issue of crime scene reconstruction has been discussed in the previous chapter but important is to define the difference between scene and scenario. The scene is a static 3D interpretation which could be observed from a variety of different angles, but it remains stable, without any movement of objects within and without any process or action is depicted. On the other hand, the scenario is the representation of process within the virtual environment which based on real area reconstruction. The possible intention of future research is utilization of connection of immersive ability of virtual scenarios and methods of scanning human physiological and psychological characteristics.

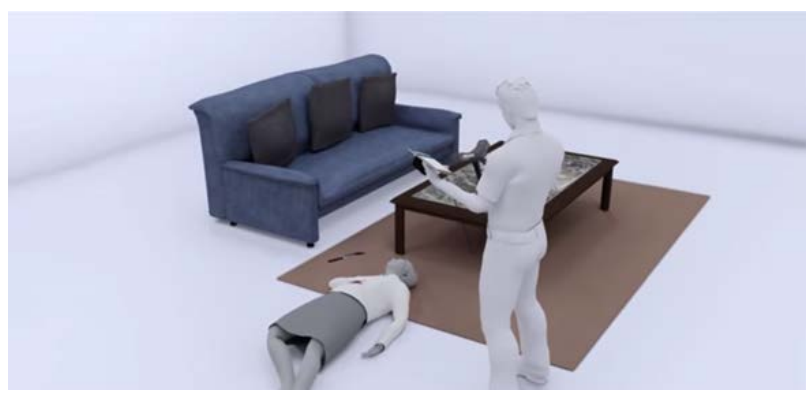

Fig. 3. Virtual crime scenario.

\section{Roles description}

It is irrelevant if the virtual environment is designed in 3D modelling software, or reconstructed based on 3D capturing technologies, creating virtual scenarios address several issues, which are relatively separated from the $3 \mathrm{D}$ reconstruction methodology. However, these techniques are defining the appropriate background for VR deployment. Highest possible immersion level of the participant is the anticipated goal of virtual scenarios; nonetheless, different roles could be taken by him within the virtual scenario. In the case of crime investigation, these roles are obvious, taken from relevant juristic and criminalistics terminology. Witness, suspect, judge or judgement commission are a typical representative of roles involved in crime investigation. For each of these individuals are valid unique approaches within 
investigation and trial, as well as in term of utilization of VR technology.

\subsection{Witness}

An allegation of the witness or even eyewitness plays a crucial role within crime commitment proving process, and crime investigation. As an amendment to standard investigation and interrogation VR scenario of the subject situation is possible to utilize to reduce bias in courtroom decision making. Another possible utilization is in witness allegation verification, where VR could be combined with methods of scanning human physiological and psychological characteristics. To reach these characteristics a variety of methods could be used, nonetheless, a polygraph, well known as lie detector was considered for the beginning phase. Other brain scanning methods like an fMRI (Functional Magnetic Resonance Imaging), EEG (Electro-Encephalography) are considered to the future research phases.

Furthermore, virtual environment, or even the whole virtual scenario design, can be constructed by the allegation of the witness. Variety of methods for virtual crime scene reconstruction should be evaluated in further research. The multi-agent system based on the simultaneous activity of several witnesses shall be used within the investigation and verification process as well. Primary the comparison of statement of individual influenced by virtual scenario represent promise future research topic. However, the first-person view and third person view is acceptable to use in case of witness immersion. The choice strictly lies on interrogating psychologist, whether is better to use a direct immersion or conservative participation in virtual scenario design process.

\subsection{Suspect}

Role of the suspect of the crime represents another category of immersed person. Relatively similar methods as in case of the witness should be realized and evaluated, however, its position is rather defensive, and this should be taken in attention within the virtual scenario design process. Relevant VR immersion techniques where the process of transition into the VR is described were published in [16]. Generally, the whole process of immersion is marginalized, but in the case of the suspect, interrogation has important value. The firstperson view is stated as a most appropriate view of immersion in case of the suspect, however also different view could be utilized in individual cases.

\subsection{Judgement commission}

An operator's view should be undertaken within the crime scene observation by judge, or commission, they supposed to be a part of whole virtual scenario creation process or at least should have access to significant facts obtained through virtual scenario design process. All gathered data should be evaluated a verified, before use within the trial. Commission could use videos, first or third person crime scene walkthroughs to get a more precise overview of the whole act.

\section{Immersion views}

The immersion view was constructed through platform Unreal engine. The Unreal 4 and Unity 3D are the most popular 3D engines nowadays. The virtual environment could be created manually through these engines. Creating the virtual world including all necessary models could be realized in a relatively simple way by drag and drop graphical user interface. The results of engines are widely utilized in computer games, but also in several VR applications. The problem in relation to the $3 \mathrm{D}$ scenario reconstruction lies in high time consumption and there are also difficulties in creating an authentic replication of real site. Nonetheless, there are some possibilities to use these engines in combination with $3 \mathrm{D}$ acquisition techniques, to make the whole process faster. Each separate view has its difficulties. The engine is still evolving nowadays, and the wide range of the modelling and $3 \mathrm{D}$ reconstruction methods can be utilized in it.

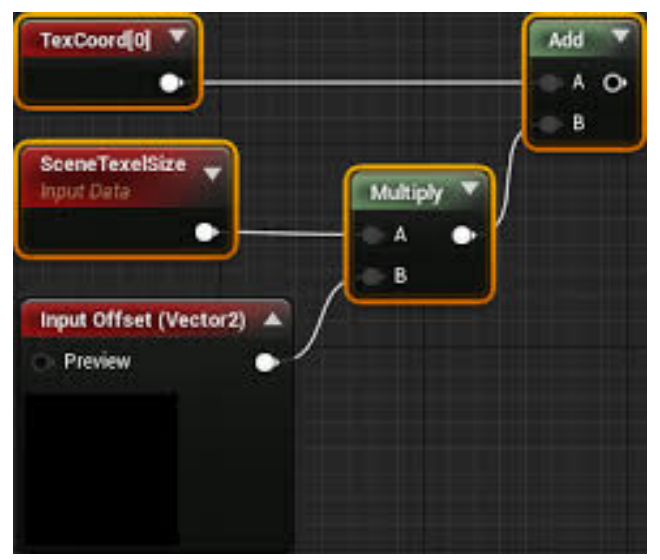

Fig. 4. Visual programing in unreal engine.

There are several major issues within the crime scene virtualization process. First is fast, precise and credible visualization and animation of environment and act participators. These could be witnesses suspects. The second one is a well-structured scenario within the environment, an order of action could influence the whole scenario with the highest weight. Another, the third important part is the incorporation of more human sensors. There are researches working on the development of VR gloves, treadmills, suits and other hardware components enhancing immersion. VR is starting to be closer to reality than the human race could imagine, and we should aware.

\section{Conclusion}

An application of virtual reality creates the wide scale of possibilities, the crime investigation improvement was proposed in the article, however, due to technology grow and setup of society we should expect diverse innovation 
in the addressed field. It is impossible to calculate the final impact of the aerial application of this visualization and immersion technology. In the other hand, we can briefly expect our future research steps. With finally purchased hardware and software, particularly, scanning machine Leica BLK 360 and software solution Capturing reality our team could start with the empiric evaluation of human virtual reality interactions.

The Acknowledgement: This work was supported by the Ministry of Education, Youth and Sports of the Czech Republic within the National Sustainability Programme Project No. LO1303 (MSMT-7778/2014) and also by the European Regional Development Fund under the project CEBIA-Tech No. CZ.1.05/2.1.00/03.0089 and by the Internal Grant Agency of Tomas Bata University under the project No. IGA/CebiaTech/2019/003.

\section{References}

1 D. Raneri, FS. (2018)

2 K. Sheppard, J.P. Cassella, S. Fieldhouse, FSI. (2017)

3 G.J. Newnham, J.D. Armston, K. Calders, M.I. Disney, J.L. Lovell, C.B. Schaaf, A.H. Strahler, F.M. Danson, RS. (2015)

4 M. Aalders, N. Adolphi, B. Daly, G. Davis, H. De Boer, S. Decker, K. Wozniak, FRI (2017)

5 U. Buck, S. Naether, B. Räss, C. Jackowski, M.J. Thali, FSI. (2013)

6 C. Villa, K. Olsen, S. Hansen, FSI. (2017)

7 A. Bornik, M. Urschler, D. Schmalstieg, H. Bischof, A. Krauskopf, T. Schwark, K. Yen, FSI. (2018)

8 D. Errickson, T.J. Thompson, B.W. Rankin, FRI 2 (3) (2014)

9 D Weiss, D McLeod-Henning, H Waltke, NIJ J (2017)

10 C.O. Young, TLR. (2014)

11 N. Salmanowitz, J. LB. 5 (1) (2018)

12 T. Kilgus, E. Heim, S. Haase, S. Prüfer, M. Müller, A. Seitel, L. Maier-Hein, IJCARS. 10 (5) (2014)

13 W. Baier, D.G. Norman, J.M. Warnett, M. Payne, N.P. Harrison, N.C. Hunt, M.A. Williams, FSI. (2017)

14 R.M. Carew, R.M. Morgan, C. Rando, JFS. (2018),

15 I Hofer, MUCL, (2007)

16 D. Sproll, J. Freiberg, T. Grechkin and B. Riecke. 3DUI (2013)/ 